\title{
The EBV lytic switch protein, Z, preferentially binds to and activates the methylated viral genome
}

\author{
Prasanna M Bhende ${ }^{1}$, William T Seaman ${ }^{1}$, Henri-Jacques Delecluse ${ }^{2} \&$ Shannon C Kenney $^{1}$
}

DNA methylation promotes gene silencing, yet the Epstein-Barr virus immediate-early protein, BZLF1 (Z), converts the virus from the latent to the lytic form of infection even when the viral genome is highly methylated. Here we show that methylation of CpG motifs in Z-responsive elements of the viral BRLF1 immediate-early promoter enhances $Z$ binding to, and activation of, this promoter. Demethylation of the viral genome impairs $Z$ activation of lytic viral genes. $Z$ is the first transcription factor that preferentially binds to, and activates, a methylated promoter. These results identify an unexpected mechanism by which Epstein-Barr virus circumvents the inhibitory effects of viral genome methylation.

Epstein-Barr virus (EBV) is a human herpesvirus that causes infectious mononucleosis and is associated with certain malignancies ${ }^{1,2}$. EBV infection of B cells usually results in latent infection. But the virus periodically reactivates and replicates in a lytic manner in a small percentage of $B$ cells. This reactivation is initiated by expression of the two immediate-early genes, BZLF1 and BRLF1 (refs. 3-8), which encode the $Z$ and $R$ proteins, respectively. Both the latent and lytic forms of infection are essential for the long-term success of this virus.

High-level expression of either EBV immediate-early protein in latently infected cells converts the virus to the lytic form. $\mathrm{Z}$ and $\mathrm{R}$ are transcriptional activators; $\mathrm{Z}$ activates expression of lytic EBV genes in essentially all EBV-positive cell lines, but $\mathrm{R}$ activates expression of lytic genes in only a subset of lines ${ }^{6,7}$. Z, a bZip protein homologous to c-fos and c-jun, binds to AP-1 and AP-1-like motifs (known as Zresponsive elements; $Z R E s)^{6,8-12}$. Together, $Z$ and $R$ induce the entire complement of early and late lytic EBV genes. If $Z$ cannot activate transcription of $B R L F 1$, however, few if any early lytic genes are transcribed, and vice versa ${ }^{6,7,11-13}$. Thus, an initial and crucial step in the switch from the latent to the lytic form of EBV infection is the ability of the two immediate-early proteins to activate one another's promoters. $\mathrm{Z}$ binds to the $B R L F 1$ promoter (Rp) at two

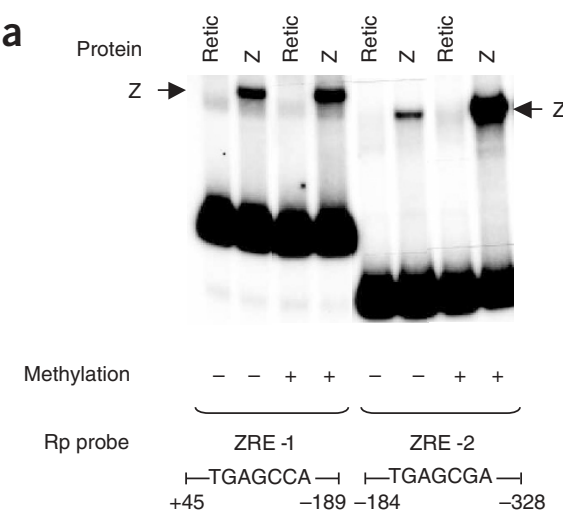

b

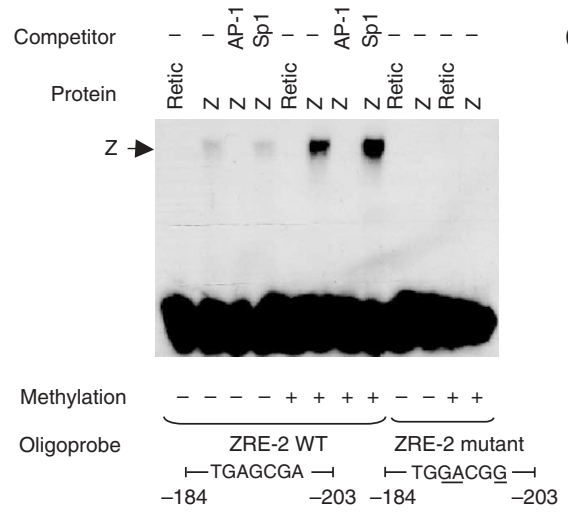

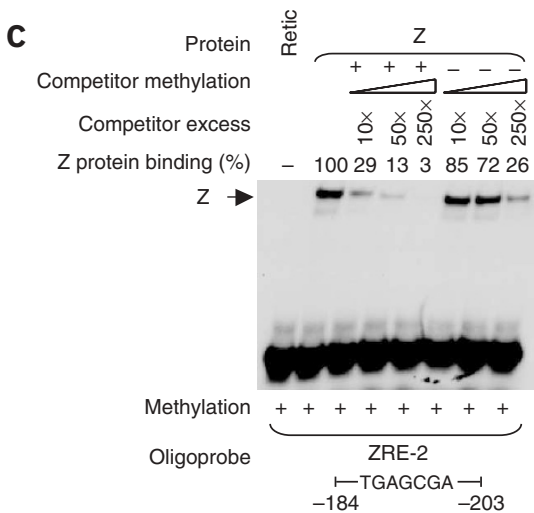

Figure $1 \mathrm{Z}$ binds preferentially to methylated ZRE-2. (a) Comparison of the binding of in vitro-translated Z to ${ }^{32} \mathrm{P}$-labeled probes spanning Rp ZRE-1 or ZRE-2, either unmethylated or methylated with SsSI CpG methylase. (b) Comparison of the binding of in vitro-translated Z to oligonucleotide probes (with or without CpG methylation) containing wild-type (WT) or mutant Rp ZRE-2. Excess unlabeled AP-1 or Sp1 competitor DNA was added as shown. (c) Comparison of the effect of methylated versus unmethylated ZRE-2 cold competitor DNA using a methylated ${ }^{32}$ P-labeled ZRE-2 probe. Z-binding complexes were quantified and the percentage of $Z$ binding (relative to the amount in the absence of specific competitor DNA, set at $100 \%$ ) in the presence of increasing amounts of cold competitor DNA is indicated. Untranslated rabbit reticulocyte lysate was used as a negative control for each probe (Retic; a-c).

${ }^{1}$ Departments of Medicine and Microbiology and Immunology, and Lineberger Comprehensive Cancer Center, University of North Carolina at Chapel Hill, North Carolina, USA. ${ }^{2}$ Department of Virus-associated tumours, German Research Cancer Centre, Heidelberg, Germany. Correspondence should be addressed to S.C.K. (shann@med.unc.edu). 
known ZREs (ZRE-1 and ZRE-2) ${ }^{11,13,14}$, whereas R activates expression of the BZLF1 promoter (Zp) indirectly through its effects on cellular transcription factors (including ATF-2 and c-jun) binding to a CRE motif ${ }^{15}$.

DNA methylation has a crucial role in modulating expression of cellular and viral genes ${ }^{16}$. Cytosine methylation usually occurs at CpG dinucleotides and is mediated by several different cellular methyltransferases ${ }^{17}$. The EBV genome is extensively methylated in latently infected cells, and methylation of key EBV transforming genes may determine whether the virus infects cells in a transforming versus nontransforming manner ${ }^{18-21}$. In addition, methylation may suppress the constitutive activity of lytic viral genes, allowing the virus to establish latency. DNA methylation causes transcriptional repression by multiple different mechanisms. DNA methylation attracts proteins with methyl-CpG-binding domains (such as MeCP2) that specifically bind methylated $\mathrm{CpGs}^{22}$ and induce repressive modifications of the chromatin, including deacetylation and methylation of histone H3 at Lys9 (ref. 20,23,24). DNA methylation also represses transcription by directly interfering with the binding of certain transcription factors.

Given the marked effect of DNA methylation on gene expression, it is unclear how certain methylated viral or cellular genes are activated under specific circumstances. At least one virus, Frog virus 3, encodes a function that promotes efficient transcription of methylated genes ${ }^{25}$. It may likewise be advantageous for gamma herpesviruses, which often have highly methylated genomes during the persistent latent forms of infection, to encode one or more proteins capable of activating methylated viral promoters. In the case of EBV, even though Rp is highly methylated in latently infected cells, Z efficiently activates $B R L F 1$ transcription from the latent viral genome. Here we show that $\mathrm{Z}$ preferentially binds to, and activates, $\mathrm{Rp}$ when the CpG motifs in Rp ZRE-2, as well as the newly discovered Rp ZRE-3, are methylated. Furthermore, $Z$ preferentially induces lytic EBV gene transcription from the methylated form of the viral genome.

Rp contains two known ZREs (ZRE-1 and ZRE-2). ZRE-2 contains the sequence TGAGCGA and thus could be methylated. We examined the methylation status of $\mathrm{Rp}$ in five different latently infected, EBVpositive cell lines by sodium bisulfite treatment of DNA, followed by DNA sequencing, as previously described ${ }^{26}$. In each cell line examined, the CpG motif in ZRE-2 was completely methylated (data not shown). We compared the binding of $Z$ to probes containing the methylated versus unmethylated forms of ZRE-2 (Fig. 1a,b). Z bound substantially better to the methylated forms of two different ZRE-2 probes but did not bind to the sequence TGGACGG in either the methylated or the unmethylated form (Fig. 1b), indicating that $Z$ does not bind to all methylated CpG motifs. The methylated form of ZRE-2 competed for binding to the methylated ZRE-2 probe at least ten times more efficiently than did the unmethylated form of ZRE-2 (Fig. 1c). We also examined $\mathrm{Z}$ binding to a series of methylated and unmethylated probes spanning regions of $\mathrm{Rp}$ from -198 to -965 (data not shown). These experiments identified an additional Rp ZRE, ZRE-3 (TTCGCGA), located from -251 to -257 . Z bound only to the methylated form of ZRE-3 (Fig. 2).

We examined the ability of $Z$ to activate the methylated versus unmethylated forms of $\mathrm{Rp}$ using a construct containing the gene chloramphenicol acetyl transferase $(C A T)$ linked to Rp (Rp-CAT; Fig. 3). Before transfection, we methylated or mock-methylated the Rp-CAT vector with SssI. In DG75 cells, $Z$ activated both the unmethylated and methylated forms of Rp-CAT (Fig. 3a). Because the constitutive activity of the methylated Rp-CAT construct was a

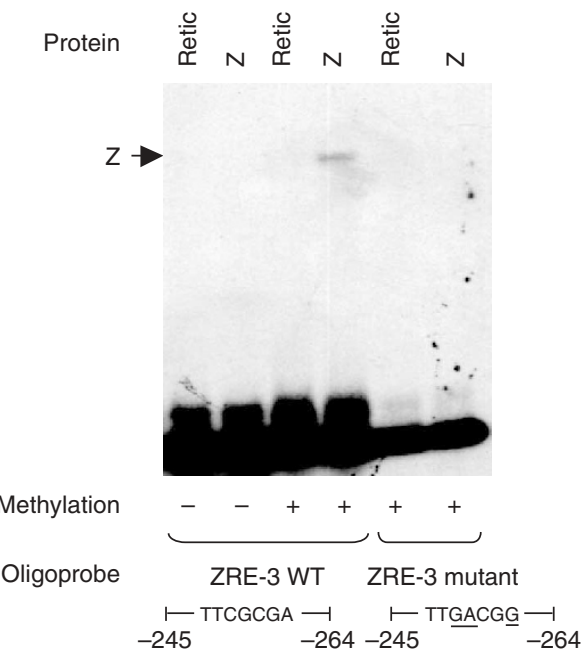

Figure $2 \mathrm{Z}$ binds only to the methylated form of a new Rp ZRE (ZRE-3). (a) Comparison of the binding of in vitro-translated $Z$ to oligonucleotide probes (with or without CpG methylation) containing wild-type (WT) or mutant Rp ZRE-3. (b) Comparison of the binding of in vitro-translated Z to ${ }^{32} \mathrm{P}$-labeled oligonucleotide probes (with or without $\mathrm{CpG}$ methylation) containing Rp ZRE-1, Rp ZRE-2, Rp ZRE-3 or the AP-1 site in the EBV $B M R F 1$ promoter. Untranslated rabbit reticulocyte lysate was used as a negative control for each probe (Retic; a,b). (c) Comparison of the binding of extracts from DG75 cells transfected with an empty vector (SG5; Vector) or a Z expression vector (SG5-Z; Z) to various probes.

b

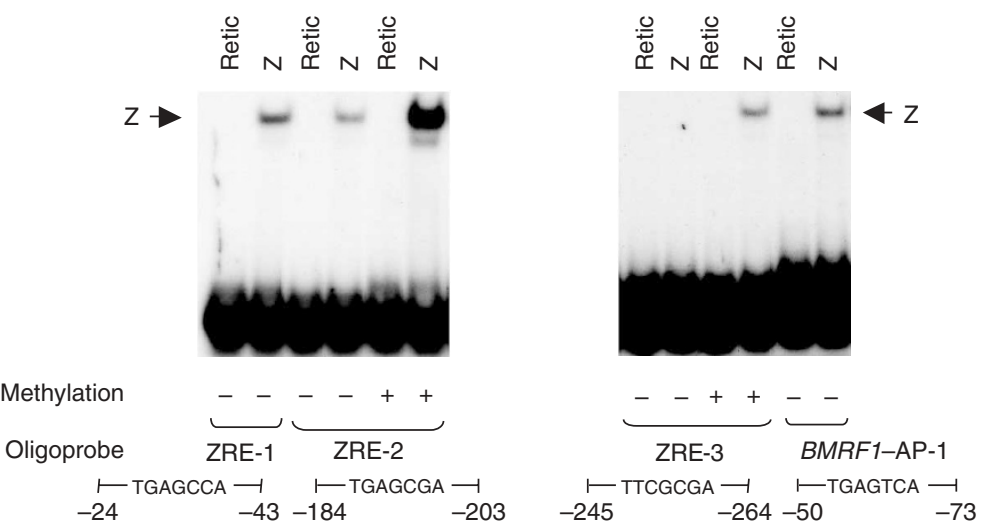

C
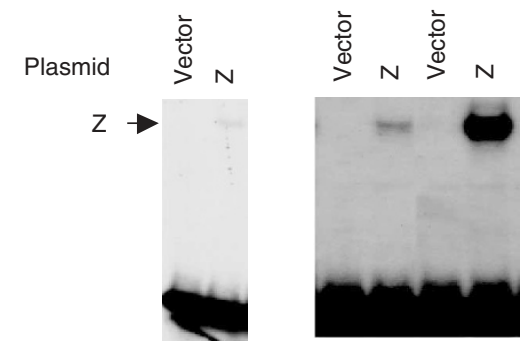

Methylation

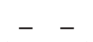

Oligoprobe

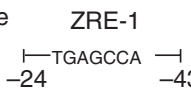

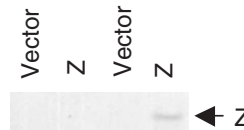

Z 
a

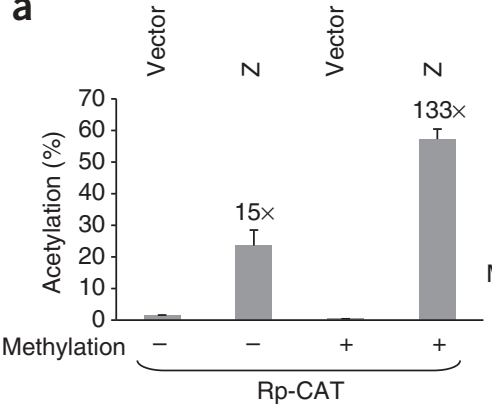

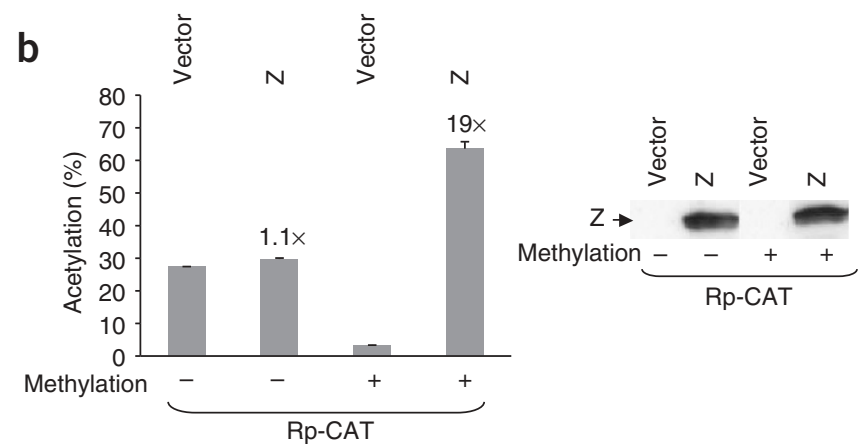

Figure 3 Z preferentially activates methylated Rp. DG75 (a) or 293T (b) cells were transfected with the Rp-CAT reporter construct (methylated or unmethylated) in combination with either the empty vector (Vector) or the $Z$ expression vector (Z). The methylation status of each promoter construct is indicated. The relative activation of the methylated versus unmethylated Rp-CAT plasmids induced by Z (relative to the effect of vector control) is indicated, in addition to the acetylation activity and the expression level of $Z$ (examined by immunoblot analysis) in each condition.

much lower than that of the unmethylated construct, however, $\mathrm{Z}$ activated the methylated form of Rp-CAT by a factor of 133 but activated the unmethylated form by a factor of only 15. In 293T cells, Z did not substantially activate the unmethylated form of Rp-CAT but activated the methylated form by a factor of $\sim 19$ (Fig. 3b). Thus, Z preferentially activates the methylated form of Rp-CAT.

$\mathrm{R}$ also induces transcription of lytic EBV genes in some, but not all, cell lines. $\mathrm{R}$ activates the two immediate-early promoters ( $\mathrm{Zp}$ and $\mathrm{Rp}$ ) indirectly through CRE (Zp) and Sp1 (Rp) motifs ${ }^{14,15}$. R activation of the Zp-CAT and Rp-CAT constructs was almost completely abrogated by vector methylation (Fig. 4a). We also ligated the methylated or unmethylated forms of Rp into the pREP4/CAT vector (after removing the RSV promoter) to create the Rp-oriP-CAT construct, which can be assembled into chromatin ${ }^{27}$. Z preferentially activated the methylated form of Rp-oriP-CAT in DG75 cells (Fig. $4 \mathbf{b}$ ), whereas R preferentially activated the unmethylated form. Prior methylation of the $\mathrm{Zp}$-CAT vector almost completely abrogated $\mathrm{Z}$ transactivation of (2) the vector (Fig. 4c). The known ZREs in Zp do not contain CpG motifs. These results suggest that the methylation status of the EBV genome in latently infected cells may differentially affect the ability of Z versus $R$ to disrupt EBV latency.

We examined the importance of the various ZREs for $\mathrm{Z}$ activation of unmethylated versus methylated Rp-CAT (Fig. 5). ZRE-1 (which does not contain a $\mathrm{CpG}$ motif) was required for $\mathrm{Z}$ activation of unmethylated Rp-CAT (Fig. 5b), as previously described ${ }^{28}$, but was completely dispensable for $\mathrm{Z}$ activation of methylated Rp-CAT in DG75 cells (Fig. 5c). In contrast, ZRE-2 and ZRE-3 were required for efficient activation of both the unmethylated and methylated forms of Rp-CAT (Fig. 5b,c). As Z does not bind detectably to unmethylated ZRE-3, a cellular factor binding to this site may be important for activation of the unmethylated promoter.

We next examined the effect of $5 \mathrm{~d}$ of treatment with 5-aza-2'deoxycytidine (a demethylating agent) in 293 cells latently infected with viruses lacking either BZLF1 or BRLF1 (ref. 12). Efficient demethylation of Rp in 293 cells treated with 5-aza-2'-deoxycytidine was confirmed by HpaII cutting (Fig. 6a), and sodium bisulfite analysis indicated that ZRE-2 was demethylated in 9 of 11 clones sequenced. In 293 cells containing a virus lacking BRLF1, prior demethylation of the viral genome enhanced $\mathrm{R}$ induction of immediate-early (Z), as well as early (BMRF1), lytic viral proteins (Fig. 6b). In contrast, demethylation of the viral genome in cells containing a virus lacking BZLF1 substantially reduced, but did not completely eliminate, $\mathrm{Z}$ activation of $B R L F 1$ and $B M R F 1$ (Fig. $6 \mathbf{c}$ ) and reduced the number of cells expressing the late viral protein virus capsid antigen (VCA) from $\sim 10 \%$ of cells to $<0.1 \%$ (Fig. 6d). These results suggest that the unique ability of $Z$ to preferentially activate the methylated form of $R p$ is important in mediating the switch from latent to lytic infection in host cells containing a methylated viral genome.

DNA methylation has a crucial role in the regulation of eukaryotic gene expression. Current models propose that DNA methylation leads
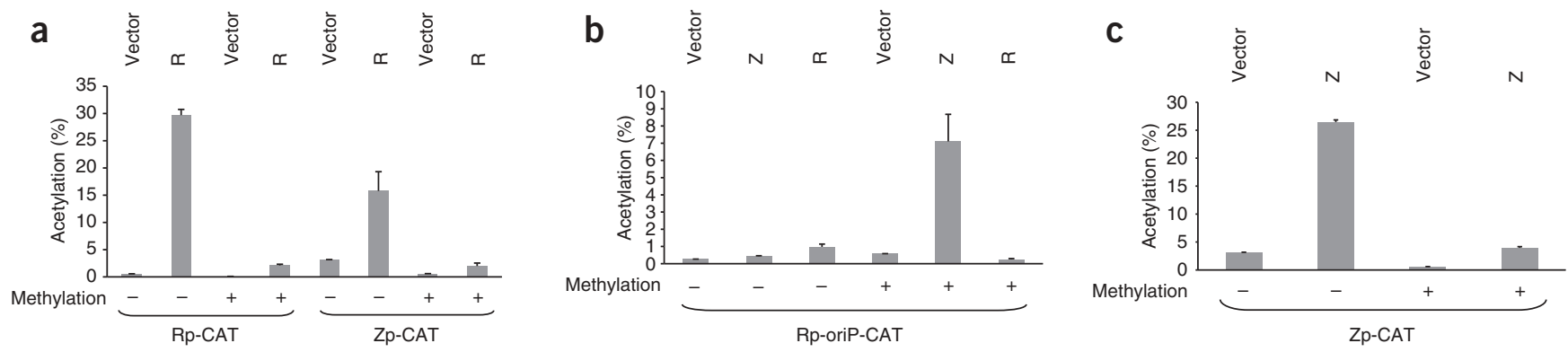

Figure 4 R preferentially activates unmethylated Rp and Zp. (a) DG75 cells were transfected with the Rp-CAT and Zp-CAT reporter constructs (methylated or unmethylated) in combination with either the empty vector (Vector) or the R expression vector (R). (b) DG75 cells were transfected with the Rp-oriP-CAT reporter construct (methylated or unmethylated) in combination with the empty vector (Vector), the $\mathrm{R}$ expression vector (R) or the $\mathrm{Z}$ expression vector (Z), and CAT activity was measured 6 d later. (c) DG75 cells were transfected with the Zp-CAT reporter construct (methylated or unmethylated) in combination with either the empty vector (Vector) or the $Z$ expression vector (Z). 
a

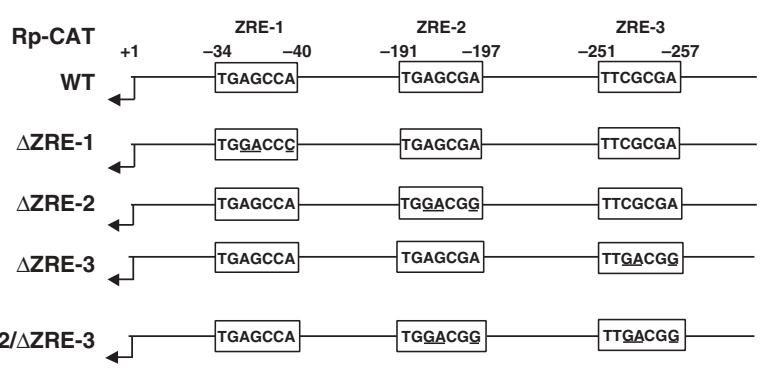

C
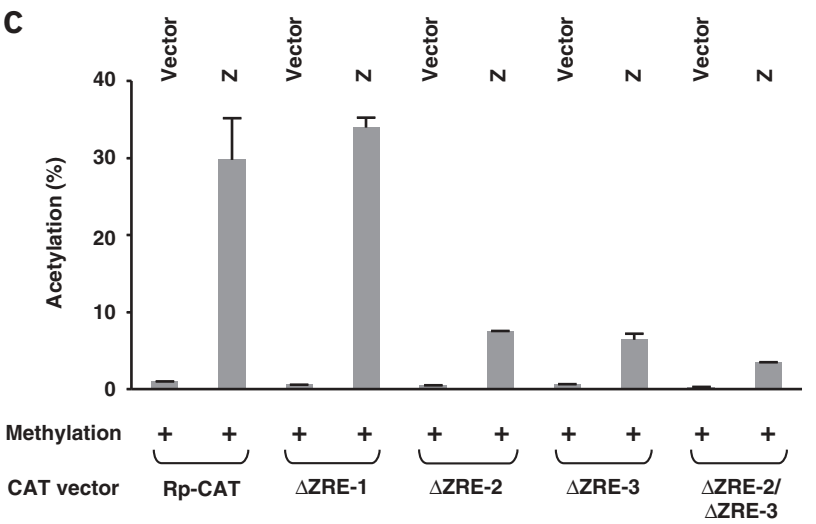

to gene silencing, either by imposing a restrictive chromatin state or by preventing the binding of transcription factors to promoters ${ }^{16,23,24}$. In sharp contrast, we show here that at least one transcription factor, the EBV Z protein, which mediates the switch between latent and lytic EBV infection, requires its immediately downstream target promoter, $\mathrm{Rp}$, to be methylated for efficient activation by $\mathrm{Z}$. We identified two ZREs in Rp that can be methylated through a CpG motif and showed that $Z$ binding to these atypical ZREs is enhanced by methylation. Furthermore, efficient $Z$ activation of Rp requires methylation of these ZREs, and the ability of $Z$ to induce transcription of lytic EBV genes in latently infected cells was dependent on the methylation state of the viral genome. The unique ability of $\mathrm{Z}$ to activate the methylated form of $\mathrm{Rp}$ may be required for the switch from the latent to the lytic form of infection in cells containing a highly methylated viral genome.

Although Rp methylation would be expected to induce a strongly inhibitory chromatin structure, the previously described ability of $Z$ to interact directly with the histone acetylase protein CBP and induce histone acetylation ${ }^{29,30}$ may overwhelm the deacetylating effects of the methyl-CpG-binding proteins. Our data suggest that whereas $\mathrm{Z}$ induces lytic EBV infection more efficiently from a methylated viral genome, $\mathrm{R}$ induces lytic infection more efficiently from an unmethylated genome. Under certain circumstances where the viral genome is not methylated (such as the completely lytic infection of normal epithelial cells), $\mathrm{R}$ may be superior to $\mathrm{Z}$ for inducing lytic gene transcription. Although $\mathrm{Rp}$ methylation enhances $\mathrm{Z}$ activation of this promoter, it probably inhibits the ability of cellular transcription factors to activate transcription of BRLF1. Thus EBV immediate-early promoter methylation could also serve to promote viral latency in situations where $\mathrm{Z}$ is limiting.

Viruses adopt new and unexpected strategies to survive in host cells under difficult conditions. In the case of EBV, viral genome methylation promotes the persistence of the virus by inhibiting the expression of viral latency proteins that are easily recognized by the host immune system, and by shutting off the constitutive expression of lytic viral

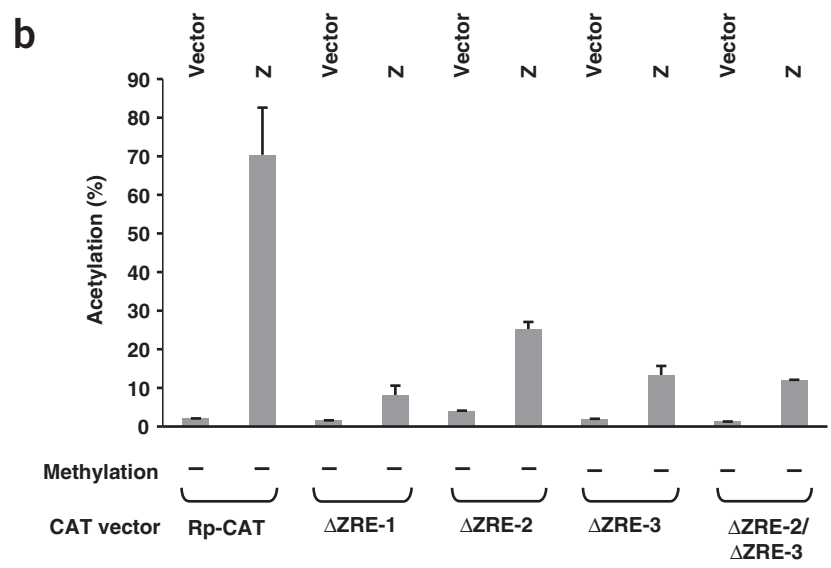

Figure 5 ZRE-2 and ZRE-3 are required for $Z$ activation of methylated Rp. (a) Site-directed mutations of the various ZREs in Rp-CAT were constructed as indicated. Specific base pairs altered are underlined. WT, wild-type. DG75 cells were transfected with the unmethylated (b) or methylated (c) $\mathrm{Rp}$-CAT constructs (wild-type versus mutant) in combination with either the empty vector (Vector) or the Z expression vector (Z).

genes. Nevertheless, the long-term success of EBV requires efficient conversion to the lytic form of infection under appropriate conditions. The unique ability of the EBV lytic switch protein $\mathrm{Z}$ to preferentially bind to, and activate, the methylated form of its downstream target gene represents a new mechanism by which a virus circumvents the inhibitory effects of DNA methylation on viral gene transcription.

\section{METHODS}

Cell lines. The 293/EBV-ZKO and 293/EBV-RKO lines are derivatives of transformed epithelial cell line 293 derived using a virus lacking BZLF1 and a virus lacking $B R L F 1$, respectively, as previously described ${ }^{12}$. DG75 is an EBVnegative Burkitt's lymphoma cell line.

Plasmids. We purified plasmid DNA through Qiagen columns as described by the manufacturer. Rp-CAT contains the immediate-early BRLF1 promoter sequences from -962 to +5 linked to the gene CAT. We created mutations in three ZREs in the Rp-CAT vector using the Quick Change Site-directed Mutagenesis kit (Stratagene). To create the Rp-oriP-CAT construct, we ligated the $B b u \mathrm{I}-\mathrm{Xba \textrm {I }}$ fragment of the methylated or unmethylated forms of Rp-CAT (which contains the Rp promoter sequences) into pREP4/CAT cut with BbuINheI (Invitrogen), removing the RSV promoter. The resultant ligated Rp-oriPCAT plasmids contain CAT driven by the methylated or unmethylated Rp and can be assembled into chromatin and replicate, as they also contain the EBV oriP element and express EBNA-1 (ref. 27). We transfected DG75 cells directly with the Rp-oriP-CAT vectors. Zp-CAT contains BZLF1 promoter sequences from -221 to +12 linked to $C A T$ (a gift from E. Flemington, Tulane University, USA). The $\mathrm{Z}$ expression vector (pSG5-Z) contains the BZLF1 gene downstream of the SV40 promoter (a gift from S.D. Hayward, Johns Hopkins University, USA) in the pSG5-vector (Stratagene). We cloned the BZLF1 cDNA (a gift from P. Farrell, Ludwig Institute for Cancer Research, London, UK) into the pSG5 vector to create $\mathrm{pSG} 5-\mathrm{ZcDNA}$ and used this vector to translate the $\mathrm{Z}$ protein in vitro. The $\mathrm{R}$ expression plasmid contains BRLF1 downstream of the SV40 promoter in the pSG5 vector (a gift from S.D. Hayward, Johns Hopkins University, USA).

Probes for electrophoretic mobility shift assay (EMSA). Rp sequences from +45 to -184 (containing ZRE-1) or from -184 to -328 (containing ZRE-2) were either left unmethylated or methylated in vitro using SssI CpG methylase 
a

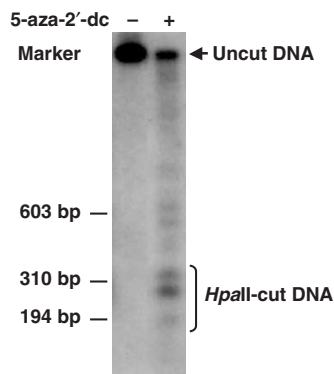

b

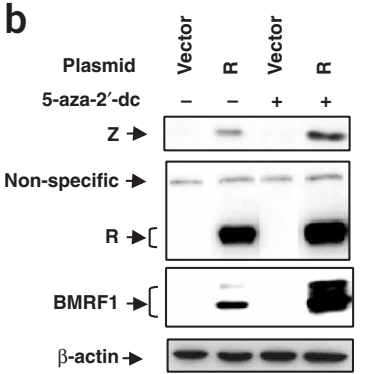

C

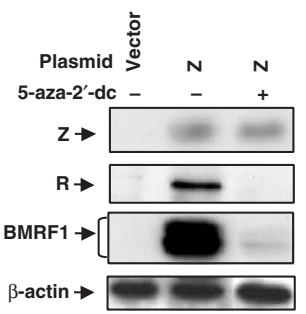

d

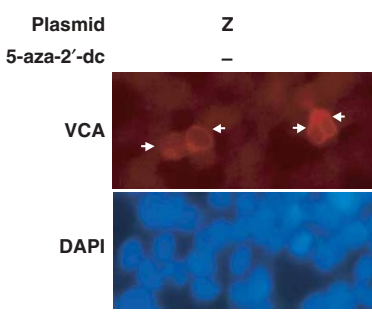

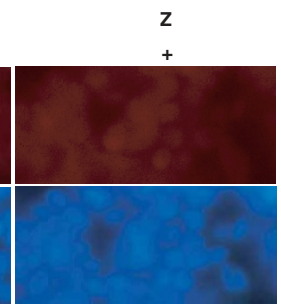

Figure $6 \mathrm{Z}$ preferentially induces expression of lytic genes from the methylated viral genome. 293 cells latently infected with a virus lacking BRLF1 (293/EBV-RKO) or BZLF1 (293/EBV-ZKO) were treated for 5 d with 5-aza-2'-deoxycytidine (5-aza-2'-dc) or left untreated. (a) The effect of 5-aza-2'deoxycytidine treatment on the methylation status of Rp in 293/EBV-ZKO cells was examined by cutting viral DNA with Hpall, which cannot cut methylated DNA, and probing with a labeled Rp probe. The expected sizes of the Hpall restriction fragments spanning the Rp probe are $326 \mathrm{bp}, 272 \mathrm{bp}$ and $190 \mathrm{bp}$. Immunoblot analysis was done on extracts from 293/EBV-RKO cells transfected with the empty vector (Vector) or the R expression vector (R; b) and on 293/ EBV-ZKO cells transfected with the empty vector (Vector) or the $Z$ expression vector $(Z ; \mathbf{c}$ ) to quantify expression of $Z$, R, BMRF1 (the early lytic EBV protein) and $\beta$-actin. (d) Expression of VCA was examined by immunofluorescence in 293/EBV-ZKO cells transfected with the $Z$ expression vector in the presence or absence of 5-aza-2'-deoxycytidine. DAPI was used to stain nuclei.

(New England Biolabs), purified by phenol-chloroform extraction and ethanol precipitation, and then labeled at the $5^{\prime}$ end with ${ }^{32} \mathrm{P}$ using the Klenow reaction. We then synthesized a series of oligonucleotides with or without methylated cytosines at the CpG motif(s) in potential ZREs (Oligos Etc.). We made synthetic oligonucleotides containing Rp ZRE-1 and AP-1 in the BMRF1 o) promoter as previously described ${ }^{11}$. We labeled synthetic double-stranded oligonucleotides at the $5^{\prime}$ end with ${ }^{32} \mathrm{P}$ using the Klenow reaction. The sequences of the oligos used in EMSAs are available on request.

EMSA. We incubated the probes either with in vitro-translated $\mathrm{Z}$ from reticulocyte lysate (or the untranslated lysate) or with whole-cell extracts made from DG75 cells electroporated with either the empty pSG5 vector or a plasmid expressing Z (pSG5-Z). We generated in vitro-translated Z protein using TNT T7 Quick Coupled Transcription/Translation System (Promega) in accordance with the manufacturer's instructions. We made DG75 extracts by suspending the cells in $200 \mu \mathrm{l}$ of a buffer containing $50 \mathrm{mM}$ HEPES (pH 7), $250 \mathrm{mM} \mathrm{NaCl}$, $0.1 \%$ Nonidet-P40, $5 \mathrm{mM}$ EDTA, $5 \mathrm{mM}$ dithiothreitol, $1 \times$ Complete Protease Inhibitors (Roche) and $15 \%$ glycerol, freeze-thawing them twice and then o centrifuging them. We used the supernatant for EMSAs. We carried out Z reactions in a buffer consisting of $100 \mathrm{mM} K C$ mM HEPES $(\mathrm{pH}$ 7.3), $10 \%$ glycerol, $0.2 \mathrm{mM}$ EDTA and $4 \mathrm{mM}$ dithiothreitol with $2 \mu \mathrm{g}$ of poly (dI-dC)/poly(dI-dC) (Pharmacia). We added $5 \mu \mathrm{g}$ of DG75 whole-cell extracts or $2 \mu \mathrm{l}$ of in vitro-translated protein to each reaction and incubated it at $4{ }^{\circ} \mathrm{C}$ for $10 \mathrm{~min}$ before adding labeled probe (10,000 c.p.m.). For competition EMSAs, we also added 10-fold, 50-fold or 250-fold excess of cold competitor DNA to the reaction before adding labeled probe. We incubated the reactions for $30 \mathrm{~min}$ at $4{ }^{\circ} \mathrm{C}$ after adding labeled probe. We then loaded the reactions onto a $4 \%$ polyacrylamide gel and separated products in $0.5 \times$ Tris-borateEDTA buffer at room temperature. We quantified the binding using a phosphorimager (Molecular Dynamics).

CAT assays. CAT vectors were mock-methylated or methylated in vitro using Sss I CpG methylase, and methylation was confirmed by complete resistance to HpaII cutting. We cleaned methylated plasmids by phenol-chloroform extraction and ethanol precipitation before using them to transfect cells. We prepared cell extracts $2 \mathrm{~d}$ (Rp-CAT or Zp-CAT vectors) or $6 \mathrm{~d}$ after transfection (Rp-oriPCAT) and incubated them at $37{ }^{\circ} \mathrm{C}$ with ${ }^{14} \mathrm{C}$-labeled chloramphenicol in the presence of acetyl coenzyme A. We determined the percent acetylation by phosphorimager screening (Molecular Dynamics).

DNA transfection. We transfected DG75 cells with DNA ( $1 \mu \mathrm{g}$ of the CAT construct plasmids and $4 \mu \mathrm{g}$ of the protein expression plasmids) by electroporation with a Zapper electroporation unit (Medical Electronics Shop, University of Wisconsin) at $1,500 \mathrm{~V}$ or a Bio-Rad electroporation unit at
$220 \mathrm{~V}$ and $975 \mu \mathrm{F}$. We resuspended all cells in RPMI 1640 medium before electroporation. We used Lipofectamine 2000 (Gibco BRL) to transfect DNA into EBV-positive 293 cells (or 293T cells) in accordance with the manufacturer's instructions.

Viral genome methylation influence on $\mathbf{Z}$ versus $\mathbf{R}$. We treated 293 cells latently infected with an EBV genome lacking BRLF1 or BZLF1 for $5 \mathrm{~d}$ in the presence or absence of 5 -aza-2'-deoxycytidine $(1 \mu \mathrm{M}$; Sigma $)$. We removed 5 -aza $2^{\prime}$-deoxycytidine during the lipofection procedure and then replaced it after $6 \mathrm{~h}$. We quantified the expression of lytic EBV proteins $2 \mathrm{~d}$ after transfection by immunoblot using antibody to BMRF1 (Capricorn), $\mathrm{R}$ (Argene) or Z (Argene).

HpaII-sensitivity assays. We either treated 293/EBV-ZKO cells with 5-aza-2'deoxycytidine or left them untreated as described above. We then collected the cells, washed them twice with $1 \times$ phosphate-buffered saline and lysed them by incubating them at room temperature for $20 \mathrm{~min}$ in a buffer containing $10 \mathrm{mM}$ Tris ( $\mathrm{pH} 7.4), 10 \mathrm{mM}$ EDTA and $0.6 \%$ SDS. We added $\mathrm{NaCl}$ to a final concentration of $1 \mathrm{M}$, incubated the lysates at $4{ }^{\circ} \mathrm{C}$ for at least $10 \mathrm{~h}$, centrifuged them $4{ }^{\circ} \mathrm{C}$ for $30 \mathrm{~min}$ and then treated the supernatants with RNase at $37{ }^{\circ} \mathrm{C}$ for $2 \mathrm{~h}$. We cleaned the DNA by phenol-chloroform extraction followed by chloroform extraction and ethanol precipitation and then carried out Southern-blot analysis. We digested $3 \mu \mathrm{g}$ of DNA with $\mathrm{HpaII}$ at $37{ }^{\circ} \mathrm{C}$ for $2 \mathrm{~h}$, separated it by electrophoresis overnight on a $1.5 \%$ agarose gel and transferred it onto nitrocellulose membrane using Turboblotter neutral transfer buffers (Schleicher and Schuell) in accordance with the manufacturer's instructions. We probed the membranes with a ${ }^{32} \mathrm{P}$-labeled DNA fragment spanning the BRLF1 promoter region from -962 to +8 using QuikHyb Hybridization Solution (Stratagene) in accordance with the manufacturer's instructions. We labeled the probe using Prime-a-Gene labeling system (Promega).

Site-directed mutagenesis. We generated Rp-CAT mutants specifically abolishing each ZRE by site-directed mutagenesis using the Quick Change Sitedirected Mutagenesis kit (Stratagene) and confirmed them by sequencing. Sequences of the oligonucleotides used to create the mutant Rp-CAT constructs are available on request.

VCA immunofluorescence. We transfected 293/EBV-ZKO cells, either untreated or treated with $1 \mu \mathrm{M} 5$-aza-2'-deoxycytidine for $5 \mathrm{~d}$, with SG5 vector or SG5-Z and then fixed them in $100 \%$ ice-cold acetone $3 \mathrm{~d}$ after transfection. We incubated cells with VCA-specific mouse monoclonal antibody (Argene) at a 1:40 dilution or an isotype control antibody and then incubated them with a 1:200 dilution of rhodamine (TRITC)-conjugated AffiniPure donkey antibody to mouse IgG ( $\mathrm{H}+\mathrm{L}$; Jackson ImmunoResearch Laboratories). We identified VCA-stained cells using fluorescence microscopy. 


\section{ACKNOWLEDGMENTS}

We thank N. Sharpless for technical assistance with bisulfite modification of DNA and B. Damania for critical review of the manuscript. This work was supported by grants from the National Institutes of Health.

\section{COMPETING INTERESTS STATEMENT}

The authors declare that they have no competing financial interests.

Received 3 March; accepted 9 August 2004

Published online at http://www.nature.com/naturegenetics/
13. Packham, G., Economou, A., Rooney, C.M., Rowe, D. \& Farrell, P.J. Structure and function of the Epstein-Barr virus BZLF1 protein. J. Virol. 64, 2110-2116 (1990).

14. Ragoczy, T. \& Miller, G. Autostimulation of the Epstein-Barr virus BRLF1 promoter is mediated through consensus Sp1 and Sp3 binding sites. J. Virol. 75, 5240-5251 (2001).

15. Adamson, A.L. et al. Epstein-Barr virus immediate-early proteins BZLF1 and BRLF1 activate the ATF2 transcription factor by increasing the levels of phosphorylated p38 and c-Jun N-terminal kinases. J. Virol. 74, 1224-1233 (2000).

16. Robertson, K. DNA methylation and chromatin - unraveling the tangled web. Oncogene 21, 5361-5379 (2002).

17. Bestor, T.H. The DNA methyltransferases of mammals. Hum. Mol. Genet. 9 2395-2402 (2000)

18. Ernberg, I. et al. The role of methylation in the phenotype-dependent modulation of Epstein-Barr nuclear antigen 2 and latent membrane protein genes in cells latently infected with Epstein-Barr virus. J. Gen. Virol. 70, 2989-3002 (1989).

19. Paulson, E.J. \& Speck, S.H. Differential methylation of Epstein-Barr virus latency promoters facilitates viral persistence in healthy seropositive individuals. J. Virol. $\mathbf{7 3}$, 9959-9968 (1999).

20. Robertson, K.D. \& Ambinder, R.F. Methylation of the Epstein-Barr virus genome in normal lymphocytes. Blood 90, 4480-4484 (1997).

21. Masucci, M.G. et al. 5-Azacytidine up regulates the expression of Epstein-Barr virus nuclear antigen 2 (EBNA-2) through EBNA-6 and latent membrane protein in the Burkitt's lymphoma line rael. J. Virol. 63, 3135-3141 (1989).

22. Hendrich, B. \& Bird, A.P. Identification and characterization of a family of mammalian methyl-CpG binding proteins. Mol. Cell. Biol. 18, 6538-6547 (1998).

23. Wade, P. Methyl CpG-binding proteins and transcriptional repression. Bioessays 23, $1131-1137$ (2001)

24. Fuks, F. et al. The methyl-CpG-binding protein MeCP2 links DNA methylation to histone methylation. J. Biol. Chem. 278, 4035-4040 (2003).

25. Thompson, J.P., Granoff, A. \& Willis, D.B. Trans-activation of a methylated adenovirus promoter by a frog virus 3 protein. Proc. Natl. Acad. Sci. USA 83, 7688-7692 (1986).

26. Herman, J.G., Graff, J.R., Myöhänen, S., Nelkin, B.D. \& Baylin, S.B. Methylationspecific PCR: A novel PCR assay for methylation status of CpG islands. Proc. Natl. Acad. Sci. USA 93, 9821-826 (1996).

27. Jenkins, P.J., Binne, U.K. \& Farrell, P.J. Histone acetylation and reactivation of Epstein-Barr virus from latency. J. Virol. 74, 710-720 (2000).

28. Liu, P. \& Speck, S.H. Synergistic autoactivation of the Epstein-Barr virus immediateearly BRLF1 promoter by Rta and Zta. Virology 310, 199-206 (2003).

29. Adamson, A. \& Kenney, S. The Epstein-Barr virus BZLF1 protein interacts physically and functionally with the histone acetylase CREB-binding protein. J. Virol. 73 , 6551-6558 (1999).

30. Deng, Z. et al. The CBP bromodomain and nucleosome targeting are required for Ztadirected nucleosome acetylation and transcription activation. Mol. Cell. Biol. 23 2633-2644 (2003) 